Max-Planck-Institut für demografische Forschung

Max Planck Institute for Demographic Research

Doberaner Strasse 114 - D-18057 Rostock - GERMANY

Tel +49 (0) 3812081 - 0; Fax +49 (0) 3812081 - 202;

http://www.demogr.mpg.de

MPIDR WORKING PAPER WP 2002-044

SEPTEMBER 2002

\title{
Alter der Kinder bei Ehescheidung der Eltern und soziale Vererbung des Scheidungsrisikos
}

\author{
Andreas Diekmann \\ Henriette Engelhardt (henriette.engelhardt@oeaw.ac.at)
}

This working paper has been approved for release by: Jan M. Hoem (hoem@ demogr.mpg.de)

Head of the Laboratory of Contemporary European Fertility and Family Dynamics.

(C) Copyright is held by the authors.

Working papers of the Max Planck Institute for Demographic Research receive only limited review.

Views or opinions expressed in working papers are attributable to the authors and do not necessarily reflect those of the Institute. 


\title{
Alter der Kinder bei Ehescheidung der Eltern und soziale Vererbung des Scheidungsrisikos ${ }^{1}$
}

\author{
Andreas Diekmann* \& Henriette Engelhardt**
}

\author{
*Institut für Soziologie \\ der Universität Bern \\ **Institut für Demographie der \\ Österreichischen Akademie der Wissenschaften \\ in Wien
}

9. September 2002

\footnotetext{
${ }^{1}$ Die empirischen Analysen zu dieser Arbeit wurden am Max-Planck-Institut für Demographische Forschung durchgeführt.
} 


\section{Einleitung und Fragestellung}

Anhand der amtlichen Statistiken der Daten aus Ehescheidungsregistern ist erkennbar, dass sich der Anteil von Ehescheidungen in der Bevölkerung über einen Zeitraum von rund hundert Jahren - von 1900 bis heute - beständig nach oben bewegt hat. Kurzfristig unterbrochen wurde der Aufwärtstrend nur durch vier historische Ereignisse, nämlich die beiden Weltkriege, die Reform des Ehescheidungsrecht 1977 in Westdeutschland und die Wiedervereinigung, die $\mathrm{zu}$ einem extremen Absinken der Scheidungszahlen in Ostdeutschland führte. Abbildung 1 vermittelt ein Bild von dieser Entwicklung.

\section{[Abbildung 1 ungefähr hier]}

Es gibt verschiedene Gründe, mit denen der säkulare Trend steigender Scheidungszahlen erklärt werden kann: Die abnehmende Stigmatisierung Geschiedener, die eigene Berufstätigkeit vieler Frauen, die damit ökonomisch nicht mehr oder nicht ausschließlich auf die Versorgung in der Ehe angewiesen sind, die verbesserten Möglichkeiten einer Wiederverheiratung oder der Gründung einer neuen Lebensgemeinschaft, der gestiegene Anteil kinderloser Ehen und auch die soziale „Vererbung des Scheidungsrisikos“ (Diekmann und Engelhardt 1995). Hinzu kommt das oftmals geäußerte Argument einer zunehmenden Überfrachtung von Ehen mit emotionalen Ansprüchen und Erwartungen, die im Alltag des Ehelebens in diesem Ausmaß nur selten oder jedenfalls nicht in allen Ehen einlösbar sind (Nave-Herz et al. 1990). Die oben erwähnten Gründe wie abnehmende Stigmatisierung, Chancen einer neuen Partnerschaft, ökonomische Unabhängigkeit eines größeren Anteils von Frauen wirken sich nicht nur auf das Scheidungsrisiko aus, sondern werden teilweise auch durch das Scheidungsrisiko vermehrt. Das Scheidungsrisiko bewegt sich damit im Sinne eines Feed-Back-Effekts selbstätig nach oben. Wir haben an anderer Stelle diese Entwicklung genauer charakterisiert und von einer „Scheidungsspirale“ gesprochen (Diekmann 1987, Diekmann 1994, Diekmann und Engelhardt 1995) ${ }^{2}$

Gedreht wird die „Scheidungsspirale“ auch von einem Faktor, der im Mittelpunkt dieser Untersuchung steht, nämlich von der intergenerationalen Transmission von Scheidungsrisiken. Kinder, die die Scheidung ihrer Eltern erlebt haben, weisen in ihrer eigenen Ehe ein stark erhöhtes Risiko der Ehescheidung auf. Damit „vererbt“ sich das Scheidungsrisiko von Generation zu Generation. In zahlreichen Studien, die meisten davon

$2 \mathrm{Zu}$ einzelnen Elementen von Feed-Back- oder „Schneeballeffekten“ bei der Erklärung steigender Scheidungsrisiken siehe auch Davis 1984 und Heekerens 1987. Vgl. auch Beck-Gernsheim 2000. 
aus den USA, wurde dieser „Transmissionseffekt“ für eine Reihe westlicher Industrieländer sehr klar belegt (Heekerens 1987, Diekmann und Engelhardt 1995, Wagner 1997, Überblick in White 1990). Einzig für Ostdeutschland konnte der Effekt nicht nachgewiesen werden (Diefenbach 1997, 2000 und Engelhardt et al. 2002). Unklar bleibt allerdings die genaue Erklärung, aus welchen Gründen und über welche intervenierenden Variablen die Scheidung der Eltern das Scheidungsrisiko in der nachfolgenden Generation ansteigen lässt. Der Grund ist sicher nicht allein der fehlende Elternteil, denn Kinder, die in Deutschland durch den II. Weltkrieg in großer Zahl zu Halbwaisen wurden, weisen in ihrer Ehe kein erhöhtes Scheidungsrisiko auf (Diekmann und Engelhardt 1999). Ein Teil des Transmissionseffekts ist auch mit der „Vererbung“ der Religionszugehörigkeit verbunden. Katholische Ehen haben im Vergleich zu anderen Bekenntnissen ein geringeres Scheidungsrisiko, und zwar sowohl in der Elterngeneration als auch in der nachfolgenden Generation. Diese, fast triviale Verknüpfung, wird oftmals übersehen. McLanahan und Bumpass (1988) führen drei weitere, mögliche Erklärungen an, die sich nicht wechselseitig ausschließen müssen: 1. Die „Hypothese ökonomischer Deprivation“: Scheidungskinder, die mit einem Elternteil - meist mit der Mutter - oder auch in Stieffamilien aufwachsen, sind eher mit materieller Knappheit konfrontiert. Das wiederum hat Auswirkungen auf Erziehung und Schulbildung, frühes Verlassen des Elternhauses und das Eingehen früher Partnerschaften, so die Vermutung. 2. Nach der „Sozialisationshypothese“ haben Auseinandersetzungen und eheliche Konflikte, die einer Ehescheidung in der Regel vorausgehen, einen langfristig wirksamen Einfluss auf Eigenschaften und Verhalten der Kinder, die die Wahrscheinlichkeit einer dauerhaften Bindung vermindern. 3. Gemäß der "Stresshypothese“ werden Kinder aus Scheidungsfamilien frühzeitig in Erwachsenenrollen gedrängt. Sie werden eher das Elternhaus verlassen und früher heiraten als Kinder, die mit beiden Eltern aufgewachsen $\operatorname{sind}^{3}$.

Nach der Stresshypothese und der Sozialisationshypothese wäre zu erwarten, dass die Scheidung der Eltern dann einen geringeren Transmissionseffekt ausübt, wenn die Kinder noch sehr jung sind, während ältere Kinder die Scheidung der Eltern vermutlich „,bewusster“ erleben. Auf der anderen Seite werden Jugendliche und fast Erwachsene die Scheidung der Eltern besser verarbeiten können, so dass eine umgekehrt u-förmige Entwicklung des Transmissionseffekts in Abhängigkeit vom Alter des Kindes bei Scheidung der Eltern zu erwarten ist. Wir erwarten demnach einen geringeren Effekt der Scheidung der Eltern bei kleinen Kindern, einen stärkeren Effekt bei jüngeren Schulkindern und wiederum einen geringeren Effekt bei den Jugendlichen und jungen Erwachsenen.

Neben der zentralen Fragestellung nach der Altersabhängigkeit des Transmissionseffekts interessiert uns, ob der früher erzielte Befund eines starken Transmissionseffekts im Westen

\footnotetext{
${ }^{3}$ Diekmann und Engelhardt (1995) berichten eine Differenz von unter einem Jahr beim Heiratsalter männlicher Scheidungswaisen $(24,5)$ gegenüber Ehemännern aus Familien mit beiden Eltern $(25,3)$. Bei den Ehefrauen ist praktisch keine Differenz feststellbar. Der „Früheheneffekt“ kann somit nur einen kleinen Anteil am Transmissionseffekt erklären.
} 
und der Abwesenheit dieses Effekts im Osten reproduzierbar ist. Darüber hinaus werden bei der Untersuchung des Transmissionseffekt mehrere Kovariablen berücksichtigt, die mutmaßlich Einflüsse auf das Scheidungsrisiko ausüben. Dazu zählen die Kohortenzugehörigkeit, das Heiratsalter der Eheleute, Kinder, Merkmale des Elternhauses, die Art des Kennenlernens und die Konfession der Eheleute. Ergebnisse zu den Effekten dieser Merkmale auf das Scheidungsrisiko sind per se von Interesse. Im Berichtsteil werden wir die Schätzungen zu den Kovariablen abschließend kommentieren.

\section{Daten und Methode}

Die Datenbasis unserer Untersuchung ist die vierte Welle des Deutschen Familiensurveys, erhoben im Auftrag des Deutschen Jugendinstituts (DJI) im Jahre 2000. Die DJI-Studie setzt sich zusammen aus einer Querschnittstichprobe (dem "replikativen Survey") und einer Panelstichprobe (der "Panelstichprobe alle Bundesländer") (siehe Infratest 2000). Beim "replikativen Survey" handelt es sich um eine repräsentative Erhebung einer Stichprobe aus der deutschen Wohnbevölkerung im Alter von 18 bis 55 Jahren. Die Panelbefragung ist eine Wiederholungsbefragung einer Stichprobe von Personen, die bereits 1988 und 1994 interviewt wurden. Um die Repräsentativität unserer Analysen zu gewährleisten, schließen wir die Teilnehmer der Panelbefragung aus und konzentrieren uns entsprechend auf die Querschnitterhebung. Abzüglich von Aussiedlern, im Ausland geborenen Personen und Übersiedlern (von Ost nach West und von West nach Ost), die beim Zuzug älter als 20 Jahre waren, umfasst die Nettostichprobe in den alten und neuen Bundesländern zusammen 6860 Personen. Berücksichtigt werden in der Analyse die noch intakten, die durch Tod eines Partners beendeten sowie die bereits geschieden Erstehen. Ausgeschlossen wurden alle Ehen, bei denen mindestens ein Partner nicht in Erstehe lebte. Von den 3925 beidseitigen Erstehen lagen in 81 Fällen keine gültigen Angaben zum Heirats-, Trennungs- bzw. Scheidungsjahr vor. Die verbleibenden 3844 Befragten bilden die Grundlage der nachfolgenden Analysen. Von ihnen haben sich 21,3\% ( $\mathrm{N}=819)$ bis zum Befragungszeitpunkt getrennt und 17,9\% (N=688) wurden geschieden. Zur Aufschlüsselung der Stichproben für Ost- und Westdeutschland siehe Tabelle 1.

[Tabelle 1 ungefähr hier]

Abhängige Variable ist die „Ehedauer“ bzw. das anhand der Ehedauer mit der Ereignisanalyse geschätzte Risiko einer Ehescheidung. Darüber hinaus ermöglicht der Datensatz die Kontrolle zahlreicher Paar- und Individualmerkmale, die als wichtige Scheidungsdeterminanten gelten (z.B. Klein und Kopp 1999, Engelhardt 2002) und daher als Kovariablen bei der Analyse einbezogen werden können. Im Einzelnen wurden die Variablen wie folgt gebildet:

- Die Ehedauer (Heiratsdatum bis Scheidungsdatum bei geschiedenen Ehen; Heiratsdatum bis Interviewdatum bei (noch) bestehenden Ehen; Heiratsdatum bis Todesdatum des 
Partners bei Auflösung der Erstehe durch den Tod des Partners) ist in Jahren erfaßt. ${ }^{4}$ Eine Episode wird als ,zensiert“ behandelt, falls die Ehe zum Befragungszeitpunkt (1. Januar 2001) noch bestand oder durch den Tod des Partners aufgelöst wurde.

- Die Art der Herkunftsfamilie der befragten Person wird mit drei dichotomen, 0/1-kodierten Variablen (Dummy-Variablen) erfaßt: Mit einem Elternteil aufgewachsen wegen Scheidung der Eltern vor Alter 17 der befragten Person (Scheidungs-Familie), mit einem Elternteil aufgewachsen wegen Tod eines Elternteils (1-Eltern-Familie: Tod), mit einem Elternteil aufgewachsen aufgrund sonstiger Gründe (1-Eltern-Familie: sonstige) und ohne Eltern aufgewachsen (0-Eltern-Familie). Zur Referenzkategorie gehören die bis zum 16. Lebensjahr bei beiden Eltern aufgewachsenen Personen (2-Eltern-Familie).

- Das Alter bei Scheidung der Eltern wird in Jahren bzw. in den Kategorien 0 bis 2, 3 bis 5, 6 bis 10 und 11-16 Jahren erfaßt.

- Für die Heiratskohorten 1971-80, 1981-90, 1991-2000 werden drei Dummy-Variablen gebildet. Diese besitzen den Code 1 für Personen, deren Eheschliessungen in den entsprechenden Zeitraum fallen. Referenzkategorie ist die Heiratskohorte 1961-1970.

- Das Heiratsalter von Ehemann und Ehefrau wird in Jahren seit der Geburt gemessen. ${ }^{5}$

- Die Kohabitationsdauer (Datum des Zusammenziehens bis Heiratsdatum) wird in Jahren erfaßt.

- Die Konfessionen der Ehepartner werden mit drei dichotomen Variablen erfasst: Beide Ehepartner katholisch, beide konfessionslos und verschiedene Konfession. Die Referenzkategorie wird durch die beidseitig evangelischen sowie sonstigen christlichen und nicht christlichen Paare gebildet.

- Hat das Paar kirchlich geheiratet, nimmt die Dummy-Variable kirchliche Heirat den Wert 1 an, 0 sonst.

- Besucht die befragte Person mindestens ein- bis dreimal pro Monat die Kirche, nimmt die dichotome Variable aktiv gläubig den Wert 1 an.

- Ist das Paar gemischt national, so wird der Dummy-Variablen Nationalitätenmix eine 1 zugewiesen.

- Liegt die Geburt des ersten Kindes zeitlich vor der Eheschließung, wird der Variable voreheliches Kind der Wert 1 zugewiesen. Berücksichtigt wurden nur erste Kinder, die gemeinsam mit dem ersten Ehepartner gezeugt wurden.

- Die Geburt des ersten ehelichen Kindes wird als zeitabhängige Kovariable Erstes Kind berücksichtigt. Diese Kovariable ist 0 bis zum Geburtsdatum und 1 ab diesem Zeitpunkt.

- Die Schulbildung des Befragten und des Ehepartners wird in Jahren gemessen. Die Bildungsjahre werden den höchsten genannten Abschlüssen wie folgt zugeordnet: Gehe noch zur Schule: 8 Jahre; vorzeitig von der Volks- oder Hauptschule abgegangen: 8 Jahre;

\footnotetext{
${ }^{4}$ Da das Heiratsdatum nur monatsgenau vorliegt, wurde als Heiratstag jeweils der 15. des relevanten Monats festgelegt. Für das Scheidungs- oder Sterbedatum des Ehegatten, das nur jahresgenau vorliegt, wurden Tag und Monat mit dem 30. Juni bestimmt. Da das genaue Interviewdatum nicht im Datensatz enthalten ist, wurde als Stichtag der 1. Januar 2001 gewählt. Die Erhebung wurde im Zeitraum vom Mai bis Anfang November 2000 durchgeführt (Infratest 2000).

5 Frauen bzw. Männer, die angaben jünger als 14 bzw. 16 Jahren geheiratet zu haben, wurden in den empirischen Analysen ausgeschlossen.
} 
Volks-/Hauptschule, Mittlere Reife/Realschulabschluss: 10 Jahre; Fachhochschulreife: 12 Jahre; Abitur (Hochschulreife): 13 Jahre. Beim Ehepartner handelt es sich um den Schulabschluss bei Beginn der Partnerschaft und bei der befragten Person um den ersten allgemeinbildenden Schulabschluss.

- Verfügt der Vater der befragten Person über einen Abiturabschluss, so wird der dichotomen Variablen Vater Abitur der Wert 1 zugewiesen.

- Hat der Befragte keine Geschwister, dann nimmt die dichotome Variable Einzelkind den Wert 1 an.

- Die Art des Kennenlernens wird durch zwei Dummy-Variablen erfasst: 1.Kennen lernen im Bekannten- oder Freundeskreis oder durch Verwandte sowie 2. durch Schule, Ausbildung, Beruf, Hobby, Verein, Sport. Referenzkategorie bilden die Paare, die sich durch eine Anzeige, im Urlaub, in der Kneipe oder Disco oder auf sonstige Weise kennen gelernt haben.

Die Mittelwerte dieser Variablen für die Gesamtstichprobe sowie getrennt für Ost- und Westdeutschland sind Tabelle 2 zu entnehmen.

[Tabelle 2 ungefähr hier]

Da die Ehedauer bis zur Scheidung nur bei den zum Zeitpunkt der Befragung geschiedenen Ehen (nicht-zensierte Fälle) bekannt ist, nicht jedoch bei den noch existierenden Ehen (zensierte Fälle), führen herkömmliche Verfahren wie einfache Tabellenanalysen oder die multivariate Regressionsanalyse im Allgemeinen zu fehlerhaften Schätzungen. Die Verfahren der Ereignisanalyse liefern dagegen auch bei Vorliegen zensierter Beobachtungen konsistente Schätzungen der Einflußstärke von Kovariablen (siehe z.B. Diekmann und Mitter 1984; Blossfeld und Rohwer 2002).

Der Verlauf der Verheiratetenquoten in Abhängigkeit von der Ehedauer und verschiedenen Typen von Elternfamilien kann dabei mit der in der Demographie üblichen „Sterbetafelmethode“ unter Einschluss der zensierten Daten geschätzt werden. Für die multivariate Analyse unter Kontrolle der Kovariablen kommen hingegen so genannte parametrische Modelle in Frage, bei denen die Form der Abhängigkeit des Scheidungsrisikos von der Ehedauer vorab spezifiziert wird.

Im Verlauf der Ehe weist das Scheidungsrisiko ein umgekehrt u-förmiges, sichelförmiges Muster auf. Das Scheidungsrisiko steigt zunächst an, erreicht ein Maximum und sinkt dann mit zunehmender Ehedauer ab. Das „Sichelmodell“ (Diekmann und Mitter 1984) kann diesen Verlauf mit der folgenden parametrischen Funktion relativ gut abbilden:

$$
h(t)=c t \mathrm{e}^{-t / \lambda}
$$


Hierbei ist $\mathrm{h}(\mathrm{t})$ das ehedauerabhängige Scheidungsrisiko, $\mathrm{t}$ die Ehedauer und $\mathrm{c}$ und $\lambda$ sind empirisch zu schätzende Parameter.

Geht man davon aus, dass die Kovariablen x das Risiko über den Proportionalitätsfaktor c beeinflussen, dann kann man für $\mathrm{c}$ die folgende Verknüpfung mit den Kovariablen schreiben:

$$
c=\exp \left(\beta_{0}+\beta_{1} x_{1}+\ldots \beta_{k} x_{k}\right)=\alpha_{0} \alpha_{1}^{x_{1}} \ldots \alpha_{k}^{x_{k}}
$$

$\beta$ bzw. $\alpha$ sind die empirisch zu schätzenden Effekte der Kovariablen. Bei der so gewählten Modellspezifikation informieren die geschätzten $\alpha$-Koeffizienten der Kovariablen über die „,relativen Risiken“. Der prozentuale Effekt einer unabhängigen Variablen im Sichel-Modell beträgt dann $100(\alpha-1) \%$. Dazu ein Beispiel. In Tabelle 3 beträgt die Schätzung des $\alpha-$ Effekts für das Heiratsalter der Ehefrau 0,94. Der prozentuale Effekt auf das Risiko beträgt demnach $100(0,94-1)=-6 \%$. Ein um ein Jahr höheres Heiratsalter der Ehefrau verringert damit das Scheidungsrisiko um sechs Prozent. Die $\alpha$-Effekte schätzen wir anhand der Daten des Familiensurveys. ${ }^{6}$

\section{Ergebnisse und Diskussion}

Betrachten wir zunächst die Verlaufskurven der Verheiratetenquoten für die westdeutschen Befragten, die mit der „Sterbetafelmethode“ geschätzt wurden. Sie werden in Abbildung 2 nach dem Typ der Herkunftsfamilie aufgeschlüsselt. Man sieht, dass die Verläufe „2 Eltern“ und der Verlauf für die Ehen von Personen, deren Herkunftsfamilie durch den Tod eines Elternteils aufgelöst wurde, fast deckungsgleich sind. Man muss bei der bivariaten Aufschlüsselung allerdings vorsichtig sein, denn letzterer Verlauf basiert teilweise auf den Daten von Kriegswaisen, die gleichzeitig zur älteren Kohorte gehören und schon von daher ein geringeres Scheidungsrisiko aufweisen. Aus der Grafik ist ferner ein deutlicher Transmissionseffekt zu entnehmen. Das höchste Scheidungsrisiko finden wir bei Befragten, die aus Familien stammen, die durch Scheidung aufgelöst wurden. Ganz anders ist das Muster in Ostdeutschland (Abbildung 3). Ein Transmissionseffekt ist nicht zu identifizieren. Der Verlauf für Befragte mit geschiedenen Eltern und Befragte aus „Zwei-Eltern-Familien“ ist nicht signifikant unterschiedlich, wobei die Anzahl der Befragten mit geschiedenen Eltern allerdings relativ gering ist $(\mathrm{N}=32)$. Eine Interpretation der Verläufe für die Gruppen mit noch geringeren Fallzahlen ist wenig sinnvoll. Als erstes Ergebnis können wir festhalten, dass sich in der (bivariaten) Analyse ein starker und signifikanter Transmissionseffekt in Westdeutschland zeigt, während ein solcher Effekt bei den ostdeutschen Ehen nicht nachweisbar ist.

\footnotetext{
${ }^{6}$ Die Effekte der Kovariablen werden unter Berücksichtigung zensierter Beobachtungen anhand der Daten (der erhobenen Ehedauern und der Kovariablenwerte) mit der Maximum-Likelihood Methode geschätzt. Die Maximum-Likelihood-Schätzwerte berechnen wir mit dem Programm TDA von Rohwer, wobei für die Schätzung der Effekte der zeitveränderlichen Kovariablen auf das Verfahren des Episodensplittings zurückgegriffen wird (z.B. Blossfeld und Rohwer 2002).
} 
[Abbildungen 2, 3 und 4 ungefähr hier]

Wenden wir uns nun unserer zentralen Fragestellung zu, wobei wir wegen der geringen Fallzahlen nur die Daten der westdeutschen Befragten verwenden. Abbildung 4 zeigt die geschätzten Verheiratetenquoten für die Teilstichprobe der westdeutschen Befragten mit geschiedenen Eltern. Die Verläufe wurden hier nach dem Alter bei Scheidung der Eltern aufgeschlüsselt. Überraschenderweise sind keine signifikanten Unterschiede zwischen den Verläufen der einzelnen Altersgruppen identifizierbar. Gemäß der bivariaten Analyse hat das Alter des Kindes bei Scheidung der Eltern keinen Einfluss auf die Stärke des Transmissionseffekts. Man kann es auch so formulieren. Die Scheidung der Eltern trifft Kinder aller Altersgruppen gleichermaßen bezüglich der Erhöhung des Scheidungsrisikos bei ihren eigenen Partnerschaften.

Dieses Ergebnis bleibt auch in der multivariaten Analyse erhalten. Sowohl bei der Operationalisierung der Variablen als „Alter bei Scheidung in Jahren“ als auch für die drei Altersklassen ergeben sich keine signifikanten Schätzwerte (Tabelle 3).

Hingegen zeigt sich bei den westdeutschen Ehen ein deutlicher Transmissionseffekt. Das Risiko von Kindern geschiedener Eltern, dass ihre eigene Ehe durch Scheidung endet, ist um den Faktor drei höher im Vergleich zu Personen, die mit beiden Eltern aufgewachsen sind. Das Ergebnis steht im Einklang mit früheren Untersuchungen von Diekmann und Engelhardt (1995, 1999). In Ostdeutschland ist ein solcher Effekt - ebenfalls im Einklang mit vorliegenden Untersuchungen (Diefenbach 1997, 1999 und Engelhardt et al. 2002) nicht nachweisbar (Tabelle 4).

[Tabelle 3 ungefähr hier]

[Tabelle 4 ungefähr hier]

In Ost und West wird das Scheidungsrisiko durch Kinder in der Ehe erheblich verringert (um zwei Drittel bis drei Viertel). Auch wenn ein Kind geboren wurde und die Eltern nach der Geburt heiraten (,voreheliches Kind“) sinkt das Scheidungsrisiko auf rund die Hälfte gegenüber kinderlosen Ehen.

Immer noch variiert das Scheidungsrisiko stark mit der Konfession. So ist im Westen das Scheidungsrisiko katholischer Ehen rund ein Drittel geringer im Vergleich zur Referenzgruppe, die hauptsächlich evangelische Ehen umfasst. „Katholisch“ hat selbst dann noch einen starken Effekt, wenn für „kirchliche Heirat“ kontrolliert wird. Im Osten stellen Personen ohne Konfession die größte Befragtengruppe, mit Abstand folgen evangelische 
Ehen, während katholische Ehen eine seltene Ausnahme bilden (siehe Tabelle 2). Ein signifikanter Konfessionseffekt, d.h. ein Effekt konfessionslos versus evangelisch, ist nicht feststellbar. Der Einfluss katholischer Konfession ist hier aber nicht prüfbar, da in der Stichprobe nur wenige katholische Ehen enthalten sind. Gemeinsam ist Ost und West die Richtung und Stärke des „Effekts“ kirchlicher Heirat. Ehen, die kirchlich getraut wurden, haben ein um rund 50 Prozent vermindertes Scheidungsrisiko als nur standesamtliche Ehen. Natürlich ist der Schätzwert nicht kausal zu deuten. Vielmehr sind Eheleute, die sich kirchlich trauen lassen, in der Regel religiöser und traditioneller in Werten und Einstellungen als Eheleute, die nur vor dem Standesbeamten erscheinen (zu einer etwas anderen Interpretation siehe Esser 2002).

Im Unterschied zu den von Diekmann und Engelhardt (1995, 1999) berichteten Ergebnissen finden wir weder bei geschwisterlos aufgewachsenen Personen (Einzelkinder) noch bei Ehen gemischter Nationalität ein signifikant höheres Scheidungsrisiko. Von Interesse ist noch, ob die Art des Kennenlernens einen Einfluss auf die Stabilität der Beziehung ausübt. Hier wurden drei Gruppen unterschieden. Eheleute, die sich im Bekannten- oder Freundeskreis oder bei Verwandten kennen gelernt haben. Jene, die sich in Vereinen, der Schule, im Beruf usw. kennen gelernt haben. Und die Gruppe von Ehepartnern, deren erster Kontakt per Anzeige, im Urlaub, in Kneipe oder Disco etc. erfolgt ist. Bei letzterer Gruppe ist das Scheidungsrisiko etwas höher, wobei die Differenz gegenüber Eheleuten, die sich in Schule oder Verein getroffen haben, signifikant ist (Tabelle 3). Der Tendenz nach zeigen sich ähnliche Unterschiede in der ostdeutschen Stichprobe, die aber die Signifikanzgrenze (Irrtumswahrscheinlichkeit 0,05) nicht überschreiten (Tabelle 4). Zwei Gründe könnten eine Rolle spielen, dass „,spontane“ Partnerschaften (im Durchschnitt) etwas instabiler sein mögen. Man kennt sich weniger gut als Eheleute, die sich in der Schule, im Beruf oder im Sportverein getroffen haben. Und die Wahrscheinlichkeit der Homogenität von Interessen und anderen Merkmalen ist geringer. Bekanntlich gilt die Weisheit: „Who does not meet does not mate!“ Wo man sich kennen gelernt hat, kann aber durchaus für die Qualität und Dauer der Partnerschaft einen Unterschied machen. 


\section{Literatur}

Blossfeld, Hans-Peter; Rohwer, Götz (2002) Techniques of Event History Modeling: New Approaches to Causal Analysis, $2^{\text {nd }}$ ed. Mahwah, N.J.: Lawrence Erlbaum Associates.

Beck-Gernsheim, Elisabeth (2000) Was kommt nach der Familie? Einblicke in neue Lebensformen. München: Beck.

Diefenbach, Heike (1997) Intergenerationale Scheidungstransmission in Deutschland: Relevanz und Erklärungsansätze. Zeitschrift für Rechtssoziologie 18: 88-105.

Diefenbach, Heike (2000) Intergenerationale Scheidungstransmission in Deutschland. Die Suche nach dem "missing link" zwischen Ehescheidung in der Elterngeneration und Ehescheidung in der Kindgeneration. Würzburg: Ergonverlag.

Diekmann, Andreas (1987) Determinanten des Heiratsalters und Scheidungsrisikos. Unveröffentlichte Habilitation. Universität München.

Diekmann, Andreas (1994) Hat das steigende Ehescheidungsrisiko das berufliche Engagement von Frauen befördert? Soziale Welt 45: 83-97.

Diekmann, Andreas; Engelhardt, Henriette (1995) Die soziale Vererbung des Scheidungsrisikos: Eine empirische Untersuchung der Transmissionshypothese mit dem deutschen Familiensurvey. Zeitschrift für Soziologie 24: 215-228.

Diekmann, Andreas; Engelhardt, Henriette (1999) The Social Inheritance of Divorce: Effects of Parent's Family Type in Postwar Germany. American Sociological Review 64: 783793.

Diekmann, Andreas und Peter Mitter (1984) A Comparison of the 'Sickle Function' with Alternative Stochastic Models of Divorce Rates. In: Andreas Diekmann und Peter Mitter (Hg.), Stochastic Modelling of Social Processes. Orlando: Academic Press.

Esser, Hartmut (2002) In guten wie in schlechten Tagen? Das Framing der Ehe und das Risiko zur Scheidung. Eine Anwendung und ein Test des Modells der Frame-Selektion. Kölner Zeitschrift für Soziologie und Sozialpsychologie 54: 27-63.

Engelhardt, Henriette (2002) Zur Dynamik von Ehescheidungen: Theoretische und empirische Analysen. Berlin: Duncker \& Humblot.

Engelhardt, Henriette, Heike Trappe und Jaap Dronkers (2002) Differences in Family Policy and the Intergenerational Transmission of Divorce: A Comparison between the former East and West Germany. Demographic Research 6, 11: 295-324. Online unter http://www.demographic-research.org.

Heekerens, Hans-Peter (1987) Das erhöhte Risiko der Ehescheidung. Zur intergenerationalen Scheidungs-Tradierung. Zeitschrift für Soziologie 16: 190-203.

Infratest (2000) Familie und Partnerbeziehung in der Bundesrepublik Deutschland (Familiensurvey 2000), Methodenbericht. München: Infratest.

Klein, Thomas; Kopp, Johannes, Hrsg. (1999) Scheidungsursachen aus soziologischer Sicht. Würzburg: Ergon.

Nave-Herz, Rosemarie; Daum-Jaballah, Marita; Hauser, Sylvia; Matthias, H.; Scheller, G. (2000) Scheidungsursachen im Wandel. Bielefeld: Kleine Verlag. 
Wagner, Michael (1997) Scheidung in Ost- und Westdeutschland. Zum Verhältnis vin Ehestabilität und Sozialstruktur seit den 30er Jahren. Frankfurt: Campus.

White, Lynn K. (1990) Determinants of Divorce: A Review of Research in the Eighties. Journal of Marriage and the Family 52: 904-912. 
Abbildung 1: Ehescheidungen in Deutschland pro 10.000 Einwohner

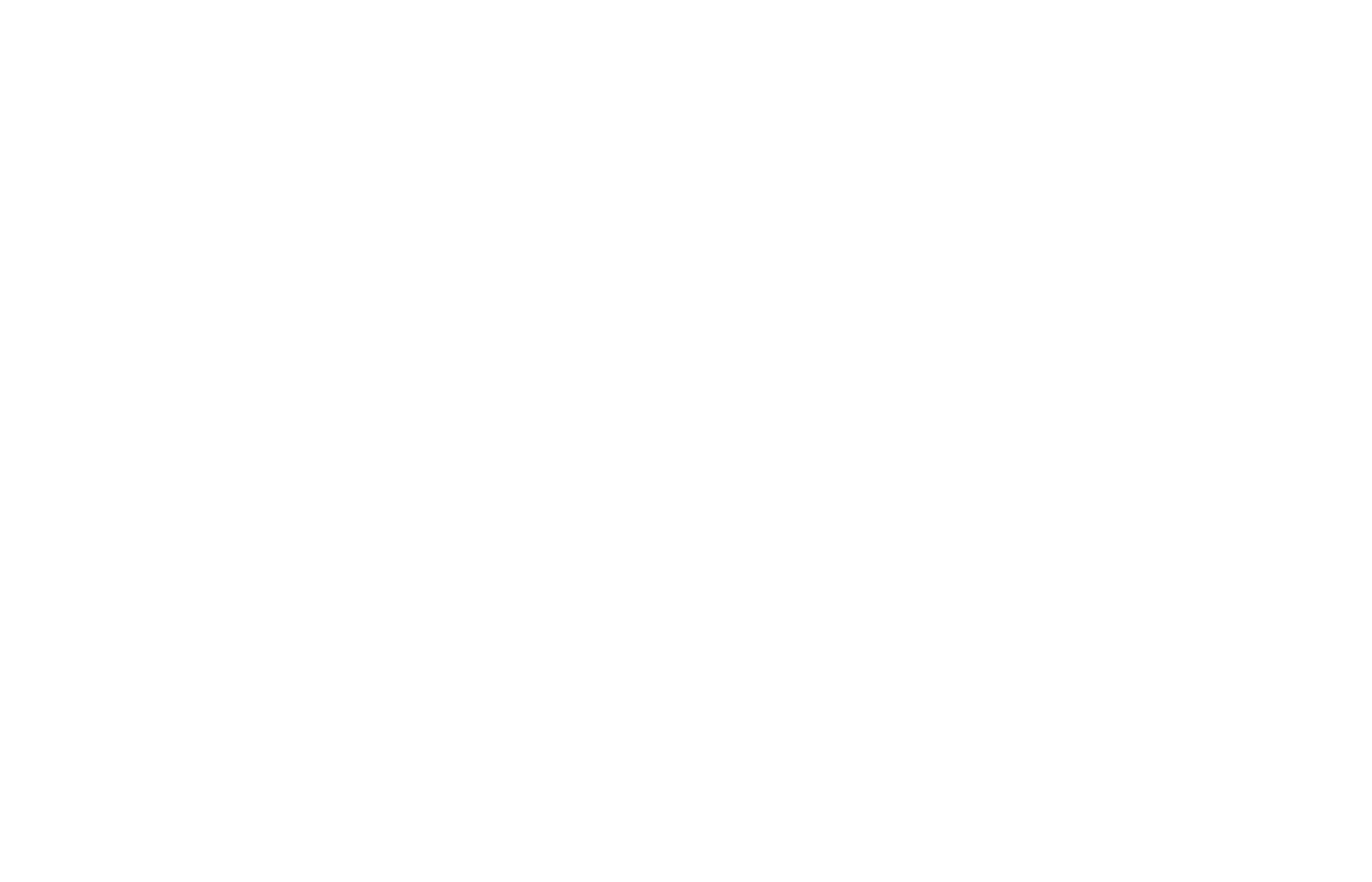


Abbildung 2: Anteil nicht geschiedener westdeutscher Erstehen nach der Ehedauer und Herkunftsfamilie

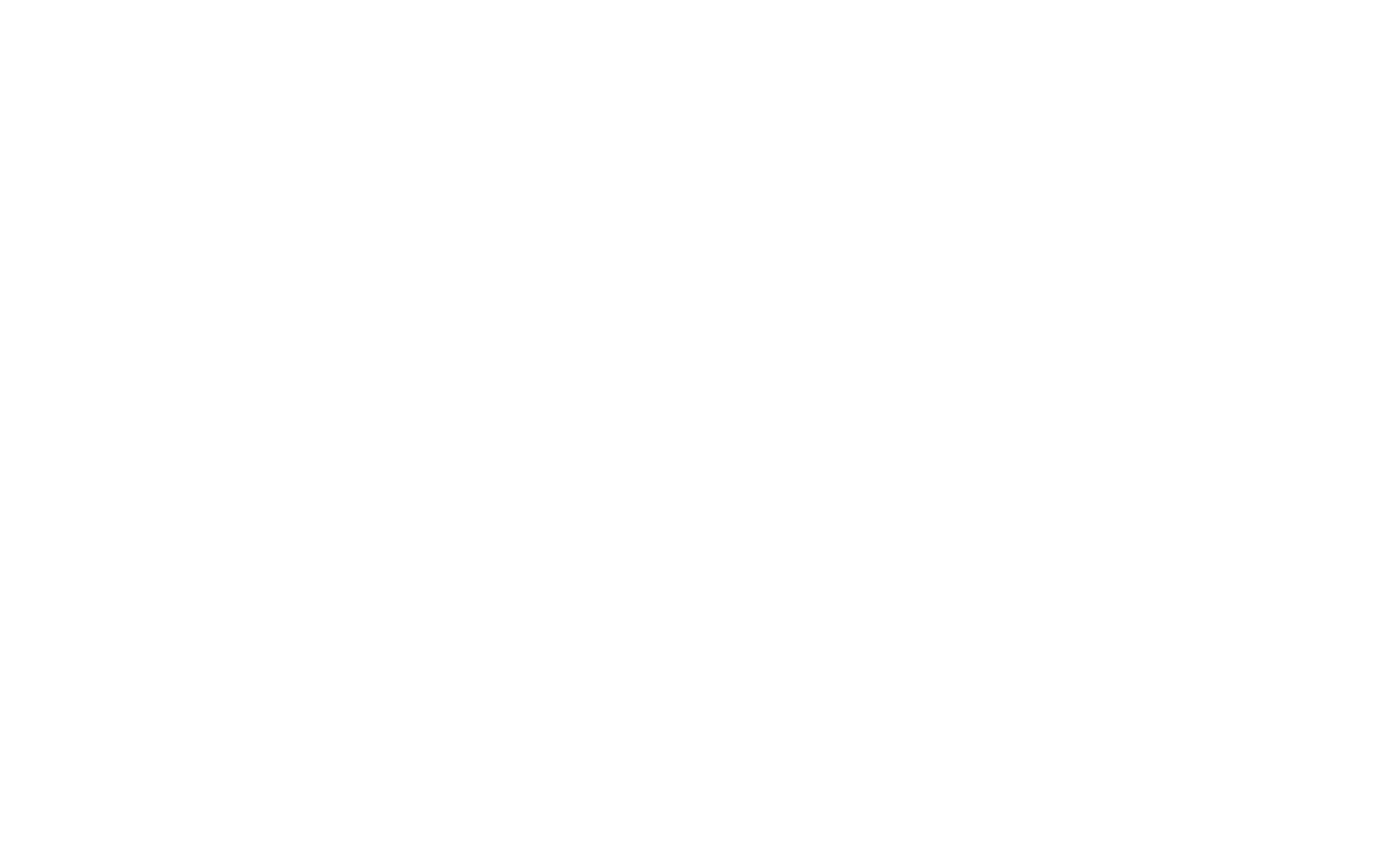

Log-Rank $($ Savage $)=0.0 ;$ Wilcoxon $($ Breslow $)=0.0$ 
Abbildung 3: Anteil nicht geschiedener ostdeutscher Erstehen nach der Ehedauer und Herkunftsfamilie

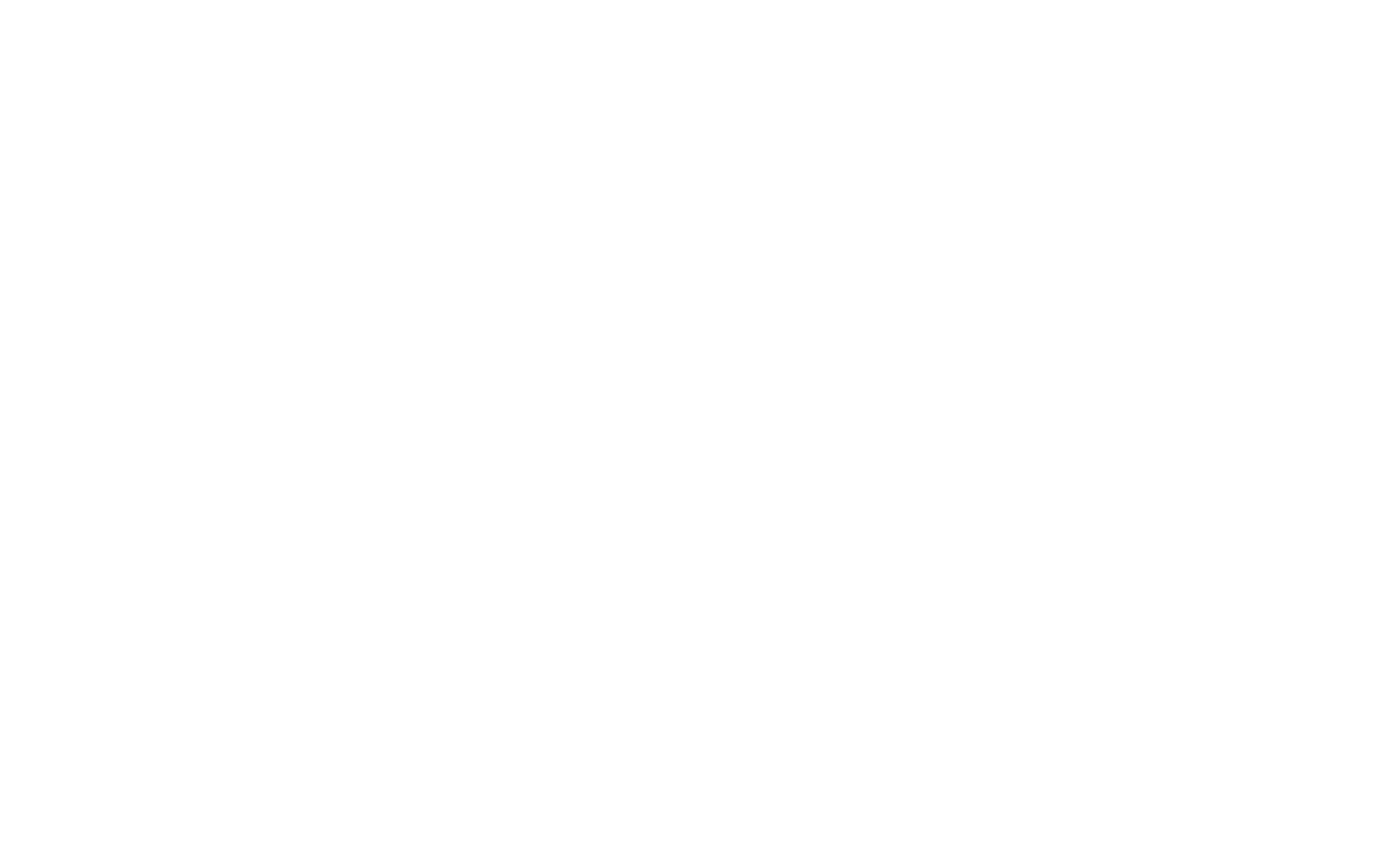

Log-Rank $($ Savage $)=0.635 ;$ Wilcoxon $($ Breslow $)=0.332$ 
Abbildung 4: Anteil nicht geschiedener westdeutscher Erstehen von Personen mit geschiedenen Eltern nach der Ehedauer und Alter bei Scheidung der Eltern

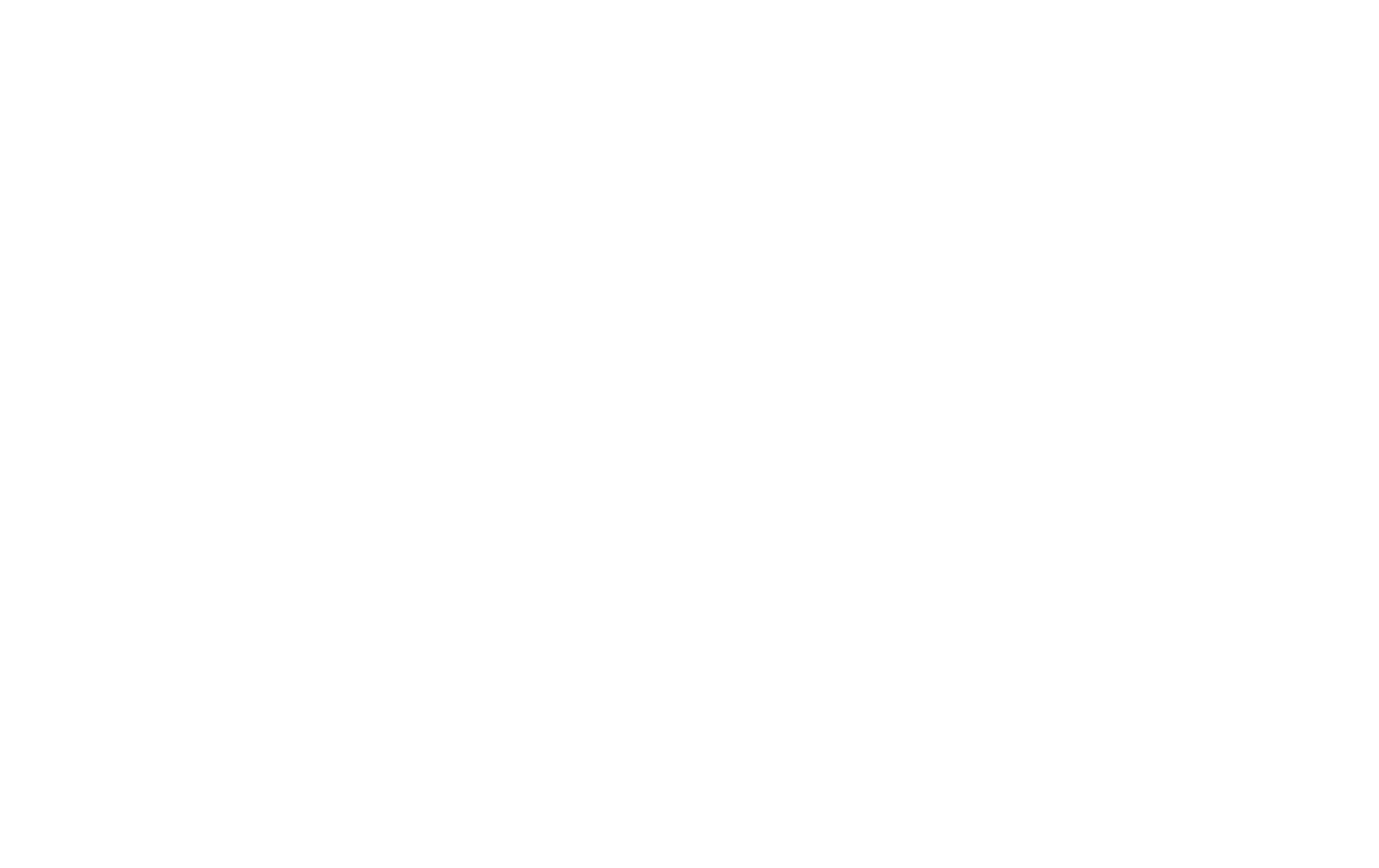

Log-Rank $($ Savage $)=0.965 ;$ Wilcoxon $($ Breslow $)=0.875$ 
Tabelle 1: Stichprobenauswahl Familiensurvey 2000

\begin{tabular}{|l|l|l|l|}
\hline & Alle & Ost & West \\
\hline Gesamtstichprobe & 10318 & 1528 & 8790 \\
Nur Querschnittstichprobe & 8091 & 1478 & 6613 \\
Deutsche Befragte & 7686 & 1461 & 6225 \\
Ohne Aussiedler, im Ausland Geborene, und Übersiedler älter 20 & 6860 & 1319 & 5541 \\
Alle Ehen & 4504 & 802 & 3702 \\
Erstehen der Befragten & 4198 & 746 & 3452 \\
Erstehen von befragter Person und Partner & 3925 & 687 & 3238 \\
Mit gültigem Heiratsjahr, Trennungsjahr, Scheidungsjahr & 3844 & 675 & 3169 \\
Getrennte Erstehen & 819 & 177 & 642 \\
Geschiedene Erstehen & 688 & 154 & 534 \\
\hline
\end{tabular}


Tabelle 2: Mittelwerte der Kovariablen

\begin{tabular}{|l|l|l|l|}
\hline & Alle & Ost & West \\
\hline 2-Eltern-Familie (0/1) & 91,4 & 90,5 & 91,6 \\
1-Eltern-Familie: Scheidung (0/1) & 4,1 & 4,8 & 4,0 \\
1-Eltern-Familie:Tod (0/1) & 1,9 & 1,4 & 2,0 \\
1-Eltern-Familie: sonstige (0/1) & 1,3 & 1,2 & 1,3 \\
0-Eltern-Familie (0/1) & 1,4 & 2,2 & 1,2 \\
Alter bei Scheidung der Eltern & 8,6 & 8,3 & 8,7 \\
\hline Heiratskohorte 1961-1970 (0/1) & 13,5 & 14,8 & 13,2 \\
Heiratskohorte 1971-1980 (0/1) & 27,8 & 35,2 & 26,2 \\
Heiratskohorte 1981-1990 (0/1) & 33,4 & 34,4 & 33,1 \\
Heiratskohorte 1991-2000 (0/1) & 25,4 & 15,6 & 27,5 \\
Heiratsalter Ehemann & 25,9 & 23,8 & 26,3 \\
Heiratsalter Ehefrau & 23,4 & 21,9 & 23,8 \\
Beziehungsalter Mann & 22,8 & 21,2 & 23,1 \\
Beziehungsalter Frau & 20,4 & 19,3 & 20,6 \\
Frau älter (0/1) & 10,2 & 8,2 & 10,7 \\
Beziehungsdauer vor HH-Gründung & 2,1 & 1,9 & 2,1 \\
Kohabitation (0/1) & 39,1 & 30,4 & 41,0 \\
Kohabitationsdauer in Jahren & 1,0 & 0,7 & 1,0 \\
Katholisches Paar (0/1) & 25,8 & 0,6 & 30,9 \\
Evangelisches Paar (0/1) & 31,0 & 13,5 & 34,6 \\
Nichtchristliches Paar (0/1) & 0,3 & 0,0 & 0,4 \\
Konfessionsloses Paar (0/1) & 11,8 & 57,9 & 2,4 \\
Verschiedene Konfession (0/1) & 31,1 & 28,0 & 31,7 \\
Verschiedene Nationalität (0/1) & 2,6 & 1,0 & 3,0 \\
Voreheliches Kind (0/1) & 12,8 & 26,6 & 9,9 \\
1. Kind in Ehe (0/1) & 69,4 & 61,4 & 71,2 \\
Schulbildung Ehemann in Jahren & 10,0 & 10,1 & 10,0 \\
Schulbildung Ehefrau in Jahren & 10,0 & 10,1 & 10,0 \\
Kirchliche Trauung (0/1) & 63,7 & 22,8 & 72,4 \\
Vater Abitur (0/1) & 8,9 & 8,8 & 9,0 \\
Einzelkind (0/1) & 19,1 & 23,6 & 18,2 \\
Kennen gelernt: Bekannte/Freunde/Verwandte (0/1) & 39,5 & 32,8 & 40,9 \\
Kennen gelernt: Schule/Beruf/Vereine (0/1) & 29,0 & 29,1 & 28,9 \\
Sonstige (Anzeige, Urlaub etc.) (0/1) & 31,5 & 38,1 & 30,1 \\
\hline \% Scheidungen & 17,9 & 22,8 & 16,8 \\
\% Trennungen & 21,3 & 26,3 & 20,3 \\
\hline Annon & & \\
\hline
\end{tabular}

Anmerkung: Die Mittelwerte der 0/1-kodierten Variablen entsprechen den Anteilwerten $\times 100$ der mit 1 kodierten Kategorien. 
Tabelle 3: Relatives Scheidungsrisiko von Personen in westdeutschen Erstehen (SichelModelle)

\begin{tabular}{|c|c|c|c|c|c|c|}
\hline & M 1 & M 2 & M 3 & M 4 & M 5 & M 6 \\
\hline 0 Eltern & $2,055^{*}$ & $2,054^{*}$ & $2,054 *$ & 1,729 & 1,798 & - \\
\hline 1 Eltern: sonstige & $1,956^{*}$ & $1,957^{*}$ & $1,957 *$ & 1,872 & 1,800 & - \\
\hline 1 Eltern: Tod & 0,901 & 0,900 & 0,900 & 1,005 & 0,967 & - \\
\hline 1 Eltern: Scheidung & $2,896 * * *$ & $3,754 * * *$ & $3,875^{* *}$ & $3,046^{*}$ & $3,183^{*}$ & $3,050 * *$ \\
\hline geschiedene Eltern*Alter bei Scheidung & & 0,975 & & & & 0,989 \\
\hline geschiedene Eltern $*$ Alter $3-5$ & & & 0,793 & 0,728 & 0,530 & \\
\hline geschiedene Eltern *Alter 6-10 & & & 0,770 & 0,868 & 0,889 & \\
\hline geschiedene Eltern *Alter 11-16 & & & 0,740 & 0,911 & 0,813 & \\
\hline Heiratskohorte 1971-1980 & & & & $1,368 *$ & $1,342^{*}$ & $1,354 *$ \\
\hline Heiratskohorte 1981-1990 & & & & $2,386 * * *$ & $2,311 * * *$ & $2,360 * * *$ \\
\hline Heiratskohorte 1991-2000 & & & & $3,100 * * *$ & $2,854 * * *$ & $2,953 * * *$ \\
\hline Heiratsalter Ehemann & & & & 0,995 & 0,988 & 0,991 \\
\hline Heiratsalter Ehefrau & & & & $0,941 * *$ & $0,940 * *$ & $0,941 * *$ \\
\hline Frau älter & & & & 1,275 & 1,159 & 1,101 \\
\hline Kohabitationsdauer & & & & 1,001 & 1,001 & 1,001 \\
\hline Katholisches Paar & & & & $0,642 * * *$ & $0,709 * *$ & \\
\hline Konfessionsloses Paar & & & & 1,192 & 0,808 & \\
\hline Verschiedene Konfession & & & & 1,194 & 1,118 & \\
\hline Verschiedene Nationalität & & & & 1,065 & 0,963 & \\
\hline Schulbildung Ehefrau & & & & 0,951 & 0,956 & 0,977 \\
\hline Schulbildung Ehemann & & & & 1,031 & 1,030 & 1,003 \\
\hline Kirchliche Heirat & & & & & $0,482 * * *$ & $0.465 * * *$ \\
\hline Einzelkind & & & & 1,078 & 1,071 & 0,988 \\
\hline Vater Abitur & & & & 1,294 & 1,220 & 1,188 \\
\hline Bekannte/Freunde/Verwandte & & & & 0,816 & 0,825 & 0,858 \\
\hline Schule/Beruf/Vereine & & & & $0,720 * *$ & $0,729 * *$ & 0,797 \\
\hline Voreheliches Kind & & & & $0,485 * * *$ & $0,441 * * *$ & $0,441 * * *$ \\
\hline 1. Kind in Ehe (zeitabhängig) & & & & $0,286 * * *$ & $0,275 * * *$ & $0,277 * * *$ \\
\hline Konstante $\alpha_{0}$ & $0,004 * * *$ & $0,004 * * *$ & $0,004 * * *$ & $0,031 * * *$ & $0,061 * * *$ & $0,055 * * *$ \\
\hline Konstante $\lambda_{0}$ & $9,018 * * *$ & $9,098 * * *$ & $9,100 * * *$ & $12,174 * * *$ & $12,575^{* * *}$ & $12,819 * * *$ \\
\hline Anzahl Episoden & 3156 & 3130 & 3130 & 3044 & 3038 & 2935 \\
\hline Anzahl Splits & & & & 5199 & 5188 & 5015 \\
\hline Anzahl Ereignisse & 532 & 525 & 525 & 497 & 496 & 478 \\
\hline -log-likelihood & 2881,232 & 2848,665 & 2048,688 & 2581,430 & 2552,168 & 2477,269 \\
\hline
\end{tabular}

Anmerkung: $\mathrm{p}<.05 ; * * \mathrm{p}<.01, * * * \mathrm{p}<.001$ (zweiseitiger Test). Referenzkategorien: 2 Eltern, Eltern nicht geschieden*Alter 0-2, Heiratskohorte 1961-70, Frau nicht älter, Evangelisches, christliches oder nicht christliches Paar, keine kichliche Heirat, kein Einzelkind, Vater kein Abitur, kennengelernt auf sonstige Weise, kein voreheliches Kind, kein eheliches Kind. In Modell 6 sind Personen, die ohne Eltern oder mit einem Elternteil aufgewachsen sind, aus der Analyse ausgeschlossen. 
Tabelle 4: Relatives Scheidungsrisiko von Personen in ostdeutschen Erstehen (SichelModelle)

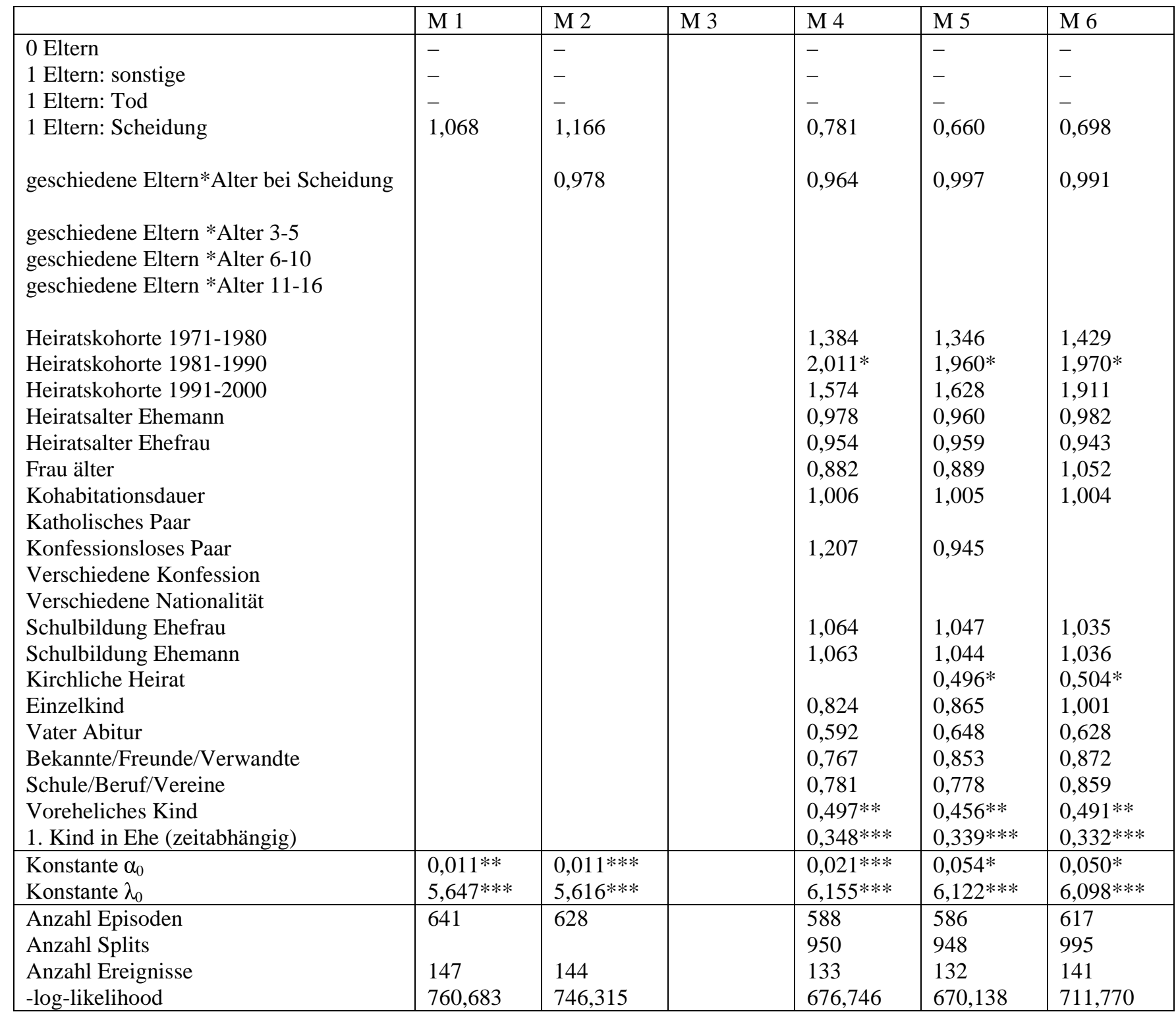

Anmerkung: $\mathrm{p}<.05 ; * * \mathrm{p}<.01, * * * \mathrm{p}<.001$ (zweiseitiger Test). Referenzkategorien: 2 Eltern, Eltern nicht geschieden*Alter 0-2, Heiratskohorte 1961-70, Frau nicht älter, einer Religionsgemeinschaft angehöriges Paar, keine kirchliche Heirat, kein Einzelkind, Vater kein Abitur, kennengelernt auf sonstige Weise, kein voreheliches Kind, kein eheliches Kind. Modell 3 wird wegen der geringen Zellenbestzung der Interaktionsterme nicht berichtet. In allen Modellen sind Personen, die ohne Eltern oder mit einem Elternteil aufgewachsen sind, aus der Analyse ausgeschlossen. 